\title{
Performance Study of Quaternion and Matrix Based Orientation for Camera Calibration
}

\author{
Rigoberto Juarez-Salazar ${ }^{1}$, Carlos Robledo-Sánchez², Fermín Guerrero-Sánchez², \\ J. Jacobo Oliveros-Oliveros ${ }^{2}$, C. Meneses-Fabian ${ }^{2}$ \\ ${ }^{1}$ División de Ingeniería Informática, Instituto Tecnológico Superior de Zacapoaxtla, \\ Zacapoaxtla, Puebla Mexico \\ ${ }^{2}$ Facultad de Ciencias Físico Matemáticas, Benemérita Universidad Autónoma de Puebla, \\ Puebla, Puebla, Mexico
}

\{Rigoberto Juarez-Salazar, rjuarezsalazar\} @ gmail.com

\begin{abstract}
The feasibility of quaternion and rotation matrix approaches to describe orientation of a camera device is presented. The usefulness of both methods is tested emphasizing its implementation for calibration of extrinsic camera parameters. Preliminary simulation results are given.
\end{abstract}

Keywords: quaternion based orientation, matrix based orientation, camera calibration.

\section{Introduction}

Camera calibration is an important task in any vision-based measurement application such as photogrammetry and optical profilometry for three-dimensional (3D) reconstruction [4]. The camera calibration procedure consists on to find the parameters of the mapping from the 3D coordinates and its corresponding two-dimensional images [5]. The calibration parameters are usually classified into intrinsic and extrinsic ones [2]. Intrinsic parameters describe internal parameters of the camera (mainly focal length, imaging distortion, and distortion center) while extrinsic parameters give information about the spatial position (x_0, y_0, z_0) and the orientation $(\gamma, \theta, \varphi)$ of the camera with respect to a reference frame as shows Fig. 1.

$3 \mathrm{D}$ matrix rotation are usually employed to describe the orientation of camera devices in most of the camera calibration methods [3]. However, unitary quaternions are very efficients to represent orientation of objects [1]. Therefore, a performance study of both quaternion- and matrix-based orientation approaches for camera calibration is addressed in this work.

In the following section, the mathematical background of both rotation matrices and unitary quaternions for rotation in three-dimensional spaces is given. Then, both approaches are evaluated by a computer simulation. The computational efficiency as well as the simplicity of implementation are emphasized. In the last section a conclusion is given. 


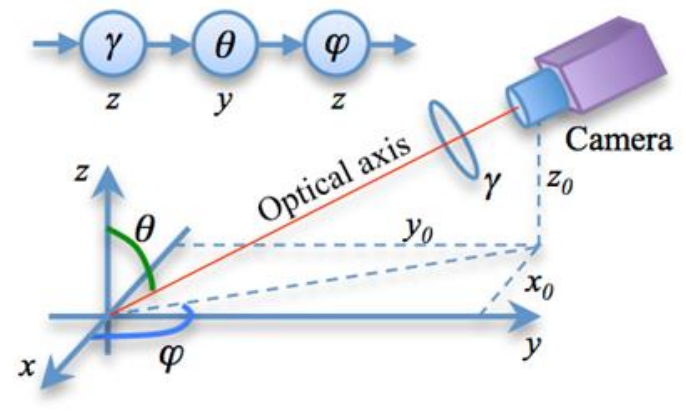

Fig. 1. Actual configuration and camera extrinsic parameters.

\section{Mathematical Background}

In the camera calibration context, the orientation (also known as angular position or attitude) consists on a set of parameters (axis and angles) which describe how a rigid body is placed in the space. In many applications, it is necessary to known or to control such parameters. Particularly, it is necessary to know or controlling the spatial and angular positions of one or more cameras for three-dimensional object reconstruction by optical methods.

For angular position, there are several mathematical tools to describe the involved parameters. For this, the most widely used are the rotation matrices and the unitary quaternions. These approaches are described below.

\subsection{Rotation Matrices}

For a given reference frame, the orientation of a rigid body can be described by setting a rotation sequence by appropriate rotation axes and the respective angles. When the rotation axes are matched with the coordinate ones, the description is known as Euler Angle-axis Sequence or simply as Euler Angles.

With this approach, it is possible to propose many angle-axis sequence and its usefulness is determined by the physical meaning or the application. In this study, we employ the following rotation sequence [see Fig. 1)]:

1. A rotation of an angle $\gamma$ about the $z$-axis,

2. A rotation of an angle $\theta$ about the $y$-axis, and

3. A rotation of an angle $\varphi$ about the z-axis.

Each of the above mentioned angle-axis operation is defined by the matrices:

$$
R_{1}(\gamma)=\left[\begin{array}{ccc}
\cos \gamma & -\sin \gamma & 0 \\
\sin \gamma & \cos \gamma & 0 \\
0 & 0 & 1
\end{array}\right]
$$




$$
\begin{aligned}
R_{2}(\theta) & =\left[\begin{array}{ccc}
\cos \theta & 0 & \sin \theta \\
0 & 1 & 0 \\
-\sin \theta & 0 & \cos \theta
\end{array}\right], \\
R_{3}(\varphi) & =\left[\begin{array}{ccc}
\cos \varphi & -\sin \varphi & 0 \\
\sin \varphi & \cos \varphi & 0 \\
0 & 0 & 1
\end{array}\right] .
\end{aligned}
$$

Thus, we have the three-dimensional rotation matrix

$$
R=R_{3}(\varphi) R_{2}(\theta) R_{1}(\gamma)
$$

\subsection{Unitary Quaternions}

Another mathematical tool to describe orientation of a rigid body is the use of unitary quaternions. Quaternions are hyper-complex numbers of the form

$$
q=q_{0}+q_{1} i+q_{2} j+q_{3} k=\left[\begin{array}{c}
q_{0} \\
\bar{q}
\end{array}\right]
$$

where $i, j, k$ are imaginary units, $q_{n}(n=1,2,3,4)$ are real numbers, $q_{0}$ and $\bar{q}=$ $\left[\begin{array}{lll}q_{1} & q_{2} & q_{3}\end{array}\right]^{T}$ are the so-called scalar and complex parts of the quaternion, respectively. Crucial for quaternions is the product rule of its imaginary units:

$$
i^{2}=j^{2}=k^{2}=i j k=-1 .
$$

By using the above product rule, the product of two quaternions $p=\left[\begin{array}{ll}p_{0} & \bar{p}^{T}\end{array}\right]^{T}$ and $q=\left[\begin{array}{ll}q_{0} & \bar{q}^{T}\end{array}\right]^{T}$ is given by

$$
p q=p_{0} q_{0}-\bar{p} \cdot \bar{q}+p_{0} \bar{q}+q_{0} \bar{p}+\bar{p} \times \bar{q},
$$

where the symbols $[\cdot]$ and $[\times]$ denote the usual vectorial inner and cross products, respectively.

For angle-axis rotation purposes in a three-dimensional space, it is convenient the notation:

$$
q=\cos \frac{\theta}{2}+u \sin \frac{\theta}{2}=\left[\begin{array}{c}
\cos \frac{\theta}{2} \\
u \sin \frac{\theta}{2}
\end{array}\right],
$$

where $u=\left[\begin{array}{lll}u_{1} & u_{2} & u_{3}\end{array}\right]^{T}$ is an unitary vector which defines the rotation axis while $\theta$ is the rotation angle about $u$. By using the quaternion in Eq. (8), the rotation of a three-dimensional vector $\bar{v}=\left[\begin{array}{lll}v_{1} & v_{2} & v_{3}\end{array}\right]^{T}$ is carried out by the quaternion product

$$
w=q v q^{*}
$$


where $v=\left[\begin{array}{cc}0 & \bar{v}^{T}\end{array}\right]^{T}$ is a pure quaternion (without scalar part) and $w=\left[\begin{array}{cc}0 & \bar{w}^{T}\end{array}\right]^{T}$ is the resulting pure quaternion where its complex vectorial part $\bar{w}$ is the rotated version of $\bar{v}$.

To obtain the involved parameters of a rotation, instead of the quaternion product in Eq. (9), a linear form is desirable. For this regard, in the following subsection is presented an alternative matrix representation for the Eq. (9).

\subsection{Matrix Description of Unitary Quaternion}

From the unitary quaternion $q$ in Eq. (8) (defining the angle and axis of the rotation), and the pure quaternion $v$ (defining the three-dimensional point to be rotated), we have for the product $q v$ :

$$
q v=\left[\begin{array}{c}
-u \cdot \bar{v} \sin \frac{\theta}{2} \\
\bar{v} \cos \frac{\theta}{2}+u \times \bar{v} \sin \frac{\theta}{2}
\end{array}\right] .
$$

Now, the rotated vector $\bar{w}$ is computed as the complex part of the resulting quaternion:

$$
w=q v q^{*}=\left[\begin{array}{c}
-u \cdot \bar{v} \sin \frac{\theta}{2} \\
\bar{v} \cos \frac{\theta}{2}+u \times \bar{v} \sin \frac{\theta}{2}
\end{array}\right]\left[\begin{array}{c}
\cos \frac{\theta}{2} \\
-u \sin \frac{\theta}{2}
\end{array}\right]=\left[\begin{array}{c}
0 \\
\bar{w}
\end{array}\right],
$$

where

$$
\bar{w}=u(u \cdot \bar{v}) \sin ^{2} \frac{\theta}{2}+\bar{v} \cos ^{2} \frac{\theta}{2}+2 u \times \bar{v} \sin \frac{\theta}{2} \cos \frac{\theta}{2}+u \times(u \times \bar{v}) \sin ^{2} \frac{\theta}{2} .
$$

By using the "vector triple product" identity, the above equation is simplified to

$$
\bar{w}=2 u(u \cdot \bar{v}) \sin ^{2} \frac{\theta}{2}+\bar{v} \cos ^{2} \frac{\theta}{2}+2 u \times \bar{v} \sin \frac{\theta}{2} \cos \frac{\theta}{2}-\bar{v} \sin ^{2} \frac{\theta}{2}
$$

For convenience, the vector inner and cross products are denoted as

$$
\begin{gathered}
u \cdot \bar{v}=u^{T} \bar{v}, \\
u \times \bar{v}=u^{\times} \bar{v},
\end{gathered}
$$

where $u^{\times}$is the skew-symmetric matrix

$$
u^{\times}=\left[\begin{array}{ccc}
0 & -u_{3} & u_{2} \\
u_{3} & 0 & -u_{1} \\
-u_{2} & u_{1} & 0
\end{array}\right] .
$$

Thus, the equation (13) can be restated as 


$$
\bar{w}=Q \bar{v},
$$

with $Q$ being the matrix

$$
Q=\cos ^{2} \frac{\theta}{2} I-\sin ^{2} \frac{\theta}{2} H+2 \sin \frac{\theta}{2} \cos \frac{\theta}{2} u^{\times},
$$

where $I$ is the identity matrix and $H$ is the Householder matrix:

$$
H=I-2 u u^{T} .
$$

From the equation (17), we can see that the rotation defined by the quaternion product $q v q^{*}$ is equivalent to the linear combination of the vector to be rotated $\bar{v}$, its Householder reflection, and the perpendicular vector between $\bar{v}$ and the rotation axis $u$.

In the following, the description of camera extrinsic parameters in an experimental configuration is given. Then, both rotation matrices and unitary quaternions to representation of the angular position are evaluated.

\section{Experimental Configuration}

The extrinsic parameters of a camera device provide a description about the physical setup as shown in Fig. 1. Particularly, by assuming a pinhole camera model, the spatial $\left(\overline{\mathrm{x}}_{0}, \overline{\mathrm{y}}_{0}, \overline{\mathrm{z}}_{0}\right)$ and angular $(\bar{\gamma}, \bar{\theta}, \bar{\varphi})$ positions of the camera device, as well as the length $\mathrm{f}$ between the pinhole and camera sensor planes, are considered.

In this work, the spatial position is not considered but rather the angular one. Accordingly, the actual configuration shown in Fig. 1 is equivalent to the case where the coordinate system is located at the camera's pinhole as shown in Fig. 2(a).

(a)

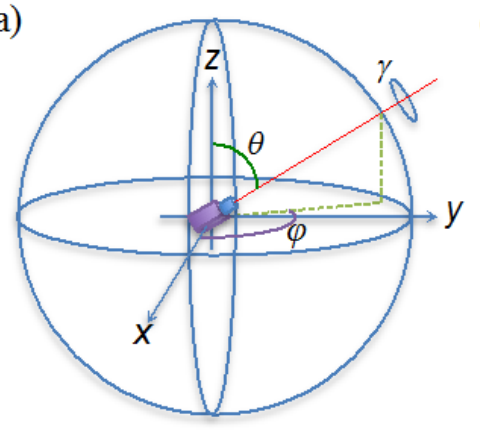

(b)

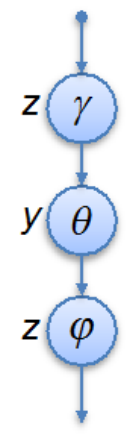

Fig. 2. (a) Angular position of a camera device. (b) Euler Angle sequence used for the matrix rotation approach. 


\section{Results}

In order to test the performance of both matrix rotation and unitary quaternion approaches, a simple computer routine was designed. This consists on perform the rotation of the camera device from an initial position. Then, such movement is simulated by computer routines.

The simplicity of implementation and the computer resources required in executing time from both approaches are compared.

The rotation considered for this study is as follows:

1. The initial position of the camera is the vertical one $[\gamma=\theta=\varphi=0$ in Fig. 2(a)] with the camera local frame aligned with the world frame.

2. A rotation is performed to align the camera's optical axis [red line in Fig. 2(a)] with the $x$ axis. Simultaneously, a rotation about the optical axis is applied from zero to $\pi / 2 \mathrm{rad}$.

The described rotation was implemented in MATLAB software. The transformation obtained from both approaches is shown in Fig. 3.

The computing time required to execute the described rotation is slightly greater for quaternion approach than matrix one. Since the computer implementation was performed on MATLAB software, a matrix-based platform, the implementation of the matrix rotation approach was direct. On the other hand, for the quaternion approach, it was necessary to define the quaternion product given by Eq. (7) and other auxiliary functions. We believe that for this reason the computing time is different.

With respect to the mathematical analysis, for camera calibration it is necessary to obtain linear forms because of the parameter estimation procedure is simpler. In this context, rotation matrix is in an appropriate format. In contrast quaternion approach requires considering an alternative matrix representation.

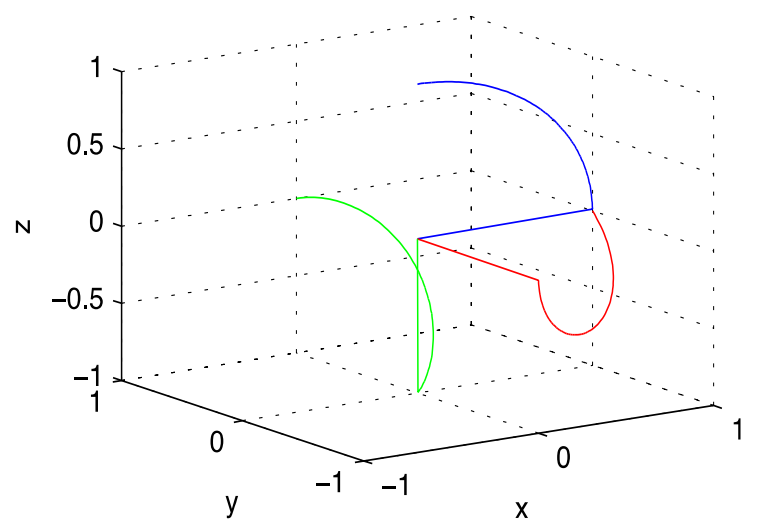

Fig. 3. Coordinates axis transformation by changing the angular position. Straigth lines denotes the unitary orthogonal vectors. Curves are the path followed by the vectors. Red, green and blue corresponds to $\mathrm{x}, \mathrm{y}$, and $\mathrm{z}$, respectively. 


\section{Conclusion}

The use of rotation matrices and unitary quaternions to describe angular position of a camera device was analyzed. For the particular case of to estimate the camera angular position, it is direct the use of rotation matrix approach while quaternion one requires additional algebraic manipulations to get an appropriate matrix format (instead of quaternion product).

Additional studies will be performed in future works to consider singularities such as gimbal lock and representation of angular position paths. This last could provide preliminary results about constrains in the solution space to make more stable the procedure of angular position with quaternions.

Acknowledgments. Authors thank the support from VIEP-BUAP, PROMEP-SEP, and CONACyT-México. R. Juarez-Salazar is grateful for the facilities provided from Instituto Tecnológico Superior de Zacapoaxtla.

\section{References}

1. Rahman, T., Krouglicof, N.: An efficient camera calibration technique offering robustness and accuracy over a wide range of lens distortion. Image Processing, IEEE Transactions on, 21(2), pp. 626-637 (2012)

2. Shah, S., Aggarwal, J.: Intrinsic parameter calibration procedure for a (high-distortion) fish-eye lens camera with distortion model and accuracy estimation. Pattern Recognition, 29(11), pp. 1775-1788 (1996)

3. Song, L. M., Wang, M. P., Lu, L., Huan, H. J.: High precision camera calibration in vision measurement. Optics \& Laser Technology, 39(7), pp. 1413-1420 (2007)

4. $\quad \mathrm{Xu}$, J., Douet, J., Zhao, J., Song, L., Chen, K.: A simple calibration method for structured light-based 3d profile measurement. Optics \& Laser Technology, 48(0), pp. 187-193 (2013)

5. Zhou, F., Cui, Y., Gao, H., Wang, Y.: Line-based camera calibration with lens distortion correction from a single image. Optics and Lasers in Engineering, 51(12), pp. 1332-1343 (2013) 